\title{
Transient Response of High Dimensional Nonlinear Dynamic System for a Rotating Cantilever Twisted Plate
}

\author{
Yuxin $\mathrm{Hao}^{1}$, Xiaojun $\mathrm{Gu}^{1}$, Wei Zhang ${ }^{2, *}$ and Jie $\mathrm{Chen}^{3}$ \\ ${ }^{1}$ College of Mechanical Engineering \& Beijing Key Laboratory of Electromechanical \\ System Measurement and Control, Beijing Information Science and Technology \\ University, Beijing 100192, China \\ 2 College of Mechanical Engineering, Beijing University of Technology, Beijing 100124, \\ China \\ ${ }^{3}$ Architecture and Civil Engineering Research Center, Shenzhen Research Institute, City \\ University of Hong Kong, Shenzhen, Guangdong 518055, China
}

Received 17 June 2019; Accepted (in revised version) 20 April 2020

\begin{abstract}
Dynamic transient responses of rotating twisted plate under the air-blast loading and step loading respectively considering the geometric nonlinear relationships are investigated using classical shallow shell theory. By applying energy principle, a novel high dimensional nonlinear dynamic system of the rotating cantilever twisted plate is derived for the first time. The use of variable mode functions by polynomial functions according to the twist angles and geometric of the plate makes it more accurate to describe the dynamic system than that using the classic cantilever beam functions and the free-free beam functions. The comparison researches are carried out between the present results and other literatures to validate present model, formulation and computer process. Equations of motion describing the transient high dimensional nonlinear dynamic response are reduced to a four degree of freedom dynamic system which expressed by out-plane displacement. The effects of twisted angle, stagger angle, rotation speed, load intensity and viscous damping on nonlinear dynamic transient responses of the twisted plate have been investigated. It's important to note that although the homogeneous and isotropic material is applied here, it might be helpful for laminated composite, functionally graded material as long as the equivalent material parameters are obtained.
\end{abstract}

AMS subject classifications: $34 \mathrm{~A} 34$

Key words: Cantilever twisted plate, rotating, blast loading, transient response, high dimensional nonlinear dynamics.

${ }^{*}$ Corresponding author.

Emails: bimhao@163.com (Y. X. Hao), guxiaojun1992@126.com (X. J. Gu), sandyzhang0@yahoo.com (W. Zhang), chenjiecnzx@sina.com (J. Chen) 


\section{Introduction}

Rotating cantilever structures, such as blades, are the key components in aero engine, turbomachine and so on. The studies of nonlinear dynamic properties of them becomes more importance because they are always subjected to various external excitation in harsh service environments which may cause severe vibration of them inevitably and even result in the complex dynamics of overall unit. One of the threats to rotating cantilever structure is blast loads because blast loads not only may make a reduction in performance and failure of the blades [1,2] but are the major safety hazard for them. In the past decade, the dynamics of the rotating cantilever structures were studied by several researchers. And in these studies rotating cantilever beams, rectangular plates, twisted plates or shallow shells were usually used.

Rosen [3] reviewed the dynamics of the pre-twisted beams and rods especially the dynamic models and solution methods. Vyas and Rao [4] derived the governing equations of motion of rotating disk-blade system and free vibrations were analyzed. Here the Coriolis forces, rotary inertia were taken into account. Lin [5] derived the governing differential equations of rotating non-uniform beam by energy principle but the Coriolis forces were not considered. To research the free vibration of pre-twisted blades, Yoo [6] applied the beam theory to obtain the motion of equations of pre-twisted blades and discussed the problem of loci veering and mode shapes variation. Chandiramani [7] researched the vibration of the anisotropic composite box-beam structure which was a simplified pre-twisted blade based on the extended Galerkin procedure. Librescu [8] studied the free vibration of functionally graded material per-twisted blades. The pertwisted blades were modeled as functionally graded material pre-twisted beams and the beams were made of metal and ceramic. Carrera et al. [9] applied finite element method and the Carrera unified formulation to rotating blades and free vibrations were analyzed. Yao [10] treated the blade as a cantilever twisted beam with pre-index angle and nonlinear dynamics of the twisted beam were studied. The chaotic dynamics were detected by numerical simulation. By using modelling method which employed hybrid deformation variables, Yoo [11] made modal analysis of the rotational pre-twisted blades by modelling method. Chortis [12] investigated the modal frequencies and damping of the beams and blades which were affected by stiffness and damping. Yang [13] established a shaft-diskblades model and the blades were simplified as beams. On the basis of Rayleigh-Ritz technique, Chen [14] computed the free vibration of the blisk with NiCrAlY coating on the blades and the system damping were also computed. Sina [15] used geometrical nonlinearities and Hamilton's principle to analyzed the vibration of composite box beams. Chen [16] employed the variational iteration to calculate the natural frequency of the rotational tapered Timoshenko beams. In above studies, the dynamics of various simple beam structures were studied.

It is reasonable for the spindling structures but if the length-width ratio of the rotating cantilever structures is small the plate or shallow shell theories will be more appropriate and the in-plane inertia should be included. Sinha [17] pointed out that Dokainish and 
Rawtani [18] solved the vibration of the rotational blade analytically by simplified them as plates first time. Then, Leissa [19] studied the free vibration of rectangular cantilever plate with certain twisted angles numerically and experimentally. Qatu [20] used the Ritz method to calculate the mode shapes and the natural frequencies of the laminated composite plates. Lim [21] presented the vibration characteristics of trapezoidal plate with certain pre-twisted angle by Ritz procedure. It had been found that because of the twisting curvature of the plate, the stretching and bending were coupled. And with the increase of twist angle the twisting frequencies would also increase. Yoo [22] performed the vibration analysis of the pre-twisted blade and there was a concentrated mass on it. $\mathrm{Hu}[23]$ proposed a numerical simulation procedure in the interests of analyzing the free vibrations of thin pre-twisted plate. Xiao [24] applied Hamilton's variational principle to derive a nonlinear dynamic equation of the thin plate and proved that there existed the dynamic softening and stiffening of the plate. Farhadi [25] studied the nonlinear dynamics of a rotating rectangular plate which was located at a rigid baffle in supersonic flow. The vibration of rotational blade was investigated by Sun [26] with the aid of the classical plate theory. Moreover, Li [27], Cao [28] and Fazelzadeh [29] researched the cantilever functionally graded material rotating plate using the plate theory. Kazanc1 [30] reviewed the response of the laminated composite plates under blast loads.

When the structures are shaped as a pre-twisted plate, it is more like a torsion shell. Recently, $\mathrm{Hu}[31,32]$ applied the shell theory to research the nonlinear vibration and linear vibration of the pre-twisted conical shell. And they thought of the elastic helicoidal shell as a twisted plate and the shell theory was used. By considering the influence of warping and the thin shell theory, Sinha $[33,34]$ presented the motion of equation in terms of transverse displacement. Qatu [35] reviewed the various laminated composite shells theory and the dynamic behaviors of shells. Sun [36] developed a new dynamic system of rotating plate with twisted angle using the shell theory.

Literature review shows that most of the studies on the dynamic of the rotating cantilever structures are focused on the free vibration and forced vibration of them subjected to periodic external excitations. It should be mentioned that as a component of the turbine, these rotating cantilever structures may be subjected to other several dynamic loading additionally, such as air blast loading, step loading and sonic boom pulses. Moreover, Nayak's research, [37], showed that the plate with cantilever boundary condition had more higher amplitude than those with SSSS (simply supported), CCCC (clamped) and CFCF (clamped-free) boundary when the plate was subjected to the transient loadings. So considering the transient dynamic response of the rotating cantilever structures subjected to blast loading is a very important problem and must be considered when they are designed.

In the present research, the transient nonlinear dynamic responses of a thin cantilever twisted plate under the air-blast loading and step loading respectively are investigated in detail. Compared with plate theory and beam theory, the shallow shell can better describe the pre-twisted characteristics of the low length-width ratio model. It is because that the cantilever twisted plate is essentially a developable ruled surface shell. One 
should present the curvature and twist curvature values of the developable ruled surface shell. Thus the shallow shell theory is applied to model the twisted plate in present. On the basis of classical shallow shell theory, the Rayleigh-Ritz method and polynomial functions, the mode shapes of the system are obtained. The comparison researches are carried out between the results presents and literatures. By using Lagrange equations and geometric nonlinear theory, high dimensional nonlinear dynamic equations of motion of the cantilever twisted plate are derived. The equations of motion describing the transient nonlinear dynamic response are analyzed by out-plane displacement for the first time. A comprehensive study about influences of twisted angle, stagger angle, rotation speed, load intensity, viscous damping and other parameters have been carried out. Although the homogeneous and isotropic material is applied in this study, it might be helpful for laminated composite, functionally graded material or sandwich twisted shell structures as long as the equivalent material parameters are obtained.

\section{Theoretical formulation}

A uniform twisted plate shown in Fig. 1 is analyzed. Four coordinates system are applied in this paper.

(a) The origin of coordinate system $O X_{1} Y_{1} Z_{1}$ is fixed in the space and the coordinate origin is at the center of the rotary hub.

(b) Coordinate system $O_{1} X Y Z$, this coordinates system is obtained by shifting $O X_{1} Y_{1} Z_{1}$ and the coordinate origin is at middle surface of the twisted plate.

(c) Body coordinate system $O_{1} x y_{0} z_{0}$, this coordinates system is set to illustrate the twist of the plate with unit vectors $(\vec{i}, \vec{j}, \vec{k})$ and the deformation of the twisted plate can be described by this coordinate system. The stagger angle of the plate is $\varphi$.

(d) Kinetic coordinate system $O_{1} x y z$, this coordinate is used to illustrate the twist of the cross-section of the plate, see [35], where $y$-axis and $y_{0}$-axis coincide at $x=0, z$-axis is normal to $x$ axes and $y$ axes at the same time. A constant twist rate $q$ is considered and twisted angle at free edge of the twisted plate in $\mathrm{x}$ direction $\theta=q a$.

It is assumed that the twisted plate characterized by span length $a$ along $x$ axes, chord length $b$ along $y$ axes and thickness $h$, respectively, is fixed on a rigid hub with radius $R$ and rotation speed $\Omega$. The twisted angle of the plate at fixed end and free end along $O_{1} x$ axis are zero and $\theta$, respectively. Thus, the position vector to an arbitrary point in middle plane of the cantilever twisted plate at orthogonal coordinate system $O_{1} x y z$ in terms of unit vectors $(\tilde{\mathbf{i}}, \tilde{\mathbf{j}}, \tilde{\mathbf{k}})$ can be expressed as

$$
\mathbf{r}_{0}=x \tilde{\mathbf{i}}+y \cos (q x) \tilde{\mathbf{j}}+y \sin (q x) \tilde{\mathbf{k}} .
$$




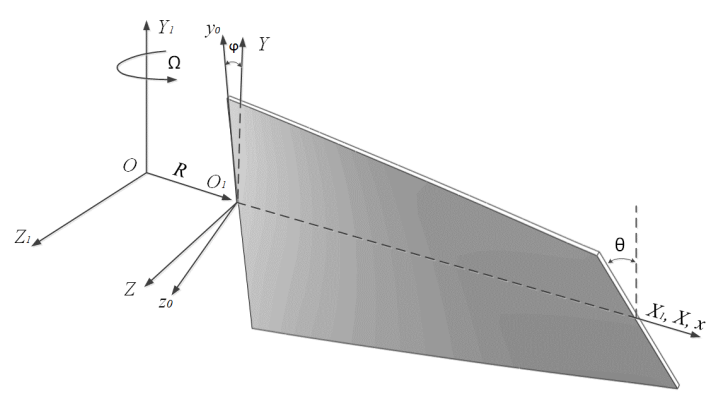

Figure 1: The model of a cantilever twisted plate and the coordinate system.

The Lame parameters for the twisted plate can be given in the Eq. (2.2) in light of differential geometry theory.

$$
\left\{\begin{array}{l}
A=\left|\mathbf{r}_{0, x}\right|=\sqrt{1+q^{2} y^{2}}, \\
B=\left|\mathbf{r}_{0, y}\right|=1 .
\end{array}\right.
$$

The unit vector $\left(\tilde{\mathbf{a}}_{1}, \tilde{\mathbf{a}}_{2}, \tilde{\mathbf{a}}_{3}\right)$ in $O_{1} x, O_{1} y$ and $O_{1} z$ direction with respect to coordinate system $O_{1} x y_{0} z_{0}$ can be written as

$$
\left[\begin{array}{c}
\tilde{\mathbf{a}}_{1} \\
\tilde{\mathbf{a}}_{2} \\
\tilde{\mathbf{a}}_{3}
\end{array}\right]=\frac{1}{A}\left[\begin{array}{ccc}
1 & -y q \sin (q x) & y q \cos (q x) \\
0 & A \cos (q x) & A \sin (q x) \\
-y q & -\sin (q x) & \cos (q x)
\end{array}\right]\left[\begin{array}{c}
\tilde{\mathbf{i}} \\
\tilde{\mathbf{j}} \\
\tilde{\mathbf{k}}
\end{array}\right] .
$$

From Eq. (2.2), one can see that the twisted plate is treated as a ruled surface shell. And the second basic homogeneous coefficients of it can be given as, see [38]

$$
\left\{\begin{array}{l}
L=\tilde{\mathbf{a}}_{3} \cdot \mathbf{r}_{0, x x}=0, \\
M=\tilde{\mathbf{a}}_{3} \cdot \mathbf{r}_{0, x y}=-\frac{q}{\sqrt{1+q^{2} y^{2}}} \\
N=\tilde{\mathbf{a}}_{3} \cdot \mathbf{r}_{0, y y}=0 .
\end{array}\right.
$$

Furthermore, the relations between radii of curvature, twist and the second fundamental form can be written as

$$
\left\{\begin{array}{l}
\frac{1}{R_{x}}=0 \\
\frac{1}{R_{y}}=0 \\
\frac{1}{R_{x y}}=\frac{M}{A B}=-\frac{q}{1+q^{2} y^{2}},
\end{array}\right.
$$

note that radii of curvature $R_{x}$ and $R_{y}$ are infinite in $x$ axes and $y$ axes, respectively, and only $R_{x y}$ is the finite radii of twist. 


\subsection{Kinematic and stress-strain relations}

Using the classical shallow shell theory, the displacement field of an arbitrary point in three directions can be expressed as

$$
\left\{\begin{array}{l}
u(x, y, z, t)=u_{0}(x, y, t)-z \frac{\partial w_{0}(x, y, t)}{\partial x} \\
v(x, y, z, t)=v_{0}(x, y, t)-z \frac{\partial w_{0}(x, y, t)}{\partial y} \\
w(x, y, z, t)=w_{0}(x, y, t)
\end{array}\right.
$$

where $u_{0}, v_{0}$ and $w_{0}$ are the displacement components of a point in the middle surface in $x, y$ and $z$ direction, respectively. On the basis of Eq. (2.6) and classical shallow shell theory, the nonlinear strain-displacement relations of twisted plate can be derived as

$$
\begin{aligned}
& \varepsilon_{x x}=\frac{\partial u}{\partial x}+\frac{w}{R_{x}}+\frac{1}{2}\left(\frac{\partial w}{\partial x}\right)^{2}, \\
& \varepsilon_{y y}=\frac{\partial v}{\partial y}+\frac{w}{R_{y}}+\frac{1}{2}\left(\frac{\partial w}{\partial y}\right)^{2} \\
& \gamma_{x y}=\frac{\partial u}{\partial y}+\frac{\partial v}{\partial x}+\frac{2 w}{R_{x y}}+\frac{\partial w}{\partial x} \frac{\partial w}{\partial y}
\end{aligned}
$$

and

$$
\varepsilon_{x x}=\varepsilon_{0 x}+z k_{x}, \quad \varepsilon_{y y}=\varepsilon_{0 y}+z k_{y}, \quad \gamma_{x y}=\gamma_{0 x y}+z k_{x y}
$$

where

$$
\begin{aligned}
& \varepsilon_{0 x}=\frac{\partial u_{0}}{\partial x}+\frac{1}{2}\left(\frac{\partial w_{0}}{\partial x}\right)^{2}, \quad \varepsilon_{0 y}=\frac{\partial v_{0}}{\partial y}+\frac{1}{2}\left(\frac{\partial w_{0}}{\partial y}\right)^{2}, \\
& \gamma_{0 x y}=\frac{\partial u_{0}}{\partial y}+\frac{\partial v_{0}}{\partial x}-\frac{2 w_{0} q}{1+q^{2} y^{2}}+\frac{\partial w_{0}}{\partial x} \frac{\partial w_{0}}{\partial y} \\
& k_{x}=-\frac{\partial^{2} w_{0}}{\partial x^{2}}, \quad k_{y}=-\frac{\partial^{2} w_{0}}{\partial y^{2}}, \quad k_{x y}=-2 \frac{\partial^{2} w_{0}}{\partial x \partial y} .
\end{aligned}
$$

With the help of Hooke's law, the stress can be expressed in terms of strain and we have

$$
\sigma_{x}=\frac{E}{1-\mu^{2}}\left(\varepsilon_{x x}+\mu \varepsilon_{y y}\right), \quad \sigma_{y}=\frac{E}{1-\mu^{2}}\left(\varepsilon_{y y}+\mu \varepsilon_{x x}\right), \quad \tau_{x y}=\frac{E}{2(1+\mu)} \gamma_{x y} .
$$

In above Eq. (2.10), $E$ is elastic modulus of the twisted plate and $\mu$ is Poisson's ratio. 


\subsection{Energy functions}

For elastic twisted plate, the strain energy can be given by

$$
\begin{aligned}
U=\iiint_{V} \frac{1}{2}\left(\sigma_{x} \varepsilon_{x x}+\sigma_{y} \varepsilon_{y y}+\tau_{x y} \gamma_{x y}\right) d V \\
=\iint_{s} \frac{1}{2}\left(\frac{E h}{1-\mu^{2}}\left(\varepsilon_{0 x}^{2}+\varepsilon_{0 y}^{2}+2 \mu \varepsilon_{0 x} \varepsilon_{0 y}\right)\right. \\
\left.\quad+\frac{E h^{3}}{12\left(1-\mu^{2}\right)}\left(k_{x}^{2}+k_{y}^{2}+2 \mu k_{x} k_{y}\right)+\frac{E h \gamma_{0 x y}^{2}}{2(1+\mu)}+\frac{E h^{3} k_{x y}^{2}}{24(1+\mu)}\right) d s .
\end{aligned}
$$

Suppose that to consider the rotational speed of the cantilever twisted plate is also $\Omega$ the same as the rigid hub since the plate is fixed on the hub. Thus, the speed of the twisted plate can be decomposed into two parts, one is the speed $\mathbf{V}_{s}$ caused by rotation of the plate and the other is the structural deformation speed $\mathbf{V}_{d}$.

After deformation, the position vector $\mathbf{r}$ at any point in the twisted plate in body coordinate system can be determined by

$$
\begin{aligned}
\mathbf{r}= & x \tilde{\mathbf{i}}+y \cos (q x) \tilde{\mathbf{j}}+y \sin (q x) \tilde{\mathbf{k}}+u \tilde{\mathbf{a}}_{1}+v \tilde{\mathbf{a}}_{2}+(w+z) \tilde{\mathbf{a}}_{3} \\
= & \left(x-\frac{z y q}{A}+\frac{u}{A}-\frac{y q w}{A}\right) \tilde{\mathbf{i}}+\left(-\frac{1}{A}(z+u y q+w) \sin (q x)+(y+v) \cos (q x)\right) \tilde{\mathbf{j}} \\
& +\left(\frac{1}{A}(z+w+u y q) \cos (q x)+(y+v) \sin (q x)\right) \tilde{\mathbf{k}} .
\end{aligned}
$$

In body coordinate system $O_{1} x y_{0} z_{0}$, the vector $\Omega$ can be written as its components and unit vectors as

$$
\mathbf{\Omega}=\Omega \cos (\varphi) \tilde{\mathbf{j}}-\Omega \sin (\varphi) \tilde{\mathbf{k}} .
$$

The velocity component vector $\mathbf{V}_{\mathbf{s}}$ caused by rotation of the plate can be presented in terms of unit vectors $(\tilde{\mathbf{i}}, \tilde{\mathbf{j}}, \tilde{\mathbf{k}})$ as follows

$$
\mathbf{V}_{s}=\mathbf{\Omega} \times \mathbf{r}=V_{x} \tilde{\mathbf{i}}+V_{y} \tilde{\mathbf{j}}+V_{z} \tilde{\mathbf{k}},
$$

where magnitude of vector components $V_{x}, V_{y}$ and $V_{z}$ can be written as

$$
\begin{aligned}
& V_{x}=\Omega\left((y+v) \sin (q x+\varphi)+\frac{1}{A}(z+y q u+w) \cos (q x+\varphi)\right), \\
& V_{y}=-\Omega\left(R+x-\frac{z y q}{A}+\frac{u}{A}-\frac{y q w}{A}\right) \sin (\varphi), \\
& V_{z}=-\Omega\left(R+x-\frac{z y q}{A}+\frac{u}{A}-\frac{y q w}{A}\right) \cos (\varphi),
\end{aligned}
$$

where $R$ is the dimension of the rigid hub. 
Deformation speed of the plate can be written as

$$
\mathbf{V}_{d}=\frac{d \mathbf{r}}{d t}=\frac{d u}{d t} \tilde{\mathbf{a}}_{1}+\frac{d v}{d t} \tilde{\mathbf{a}}_{2}+\frac{d w}{d t} \tilde{\mathbf{a}}_{3}
$$

The composed velocity $\mathbf{V}_{T}$ at any point in the twisted plate is

$$
\mathbf{V}_{T}=\mathbf{V}_{d}+\mathbf{V}_{s}
$$

Substituting the Eqs. (2.14)-(2.16) into Eq. (2.17), the composed velocity can be given as component form by

$$
\left\{\begin{array}{l}
V_{1}=\mathbf{V}_{T} \cdot \tilde{\mathbf{a}}_{1}=\dot{u}+\frac{V_{x}}{A}-\frac{y q \Omega \cos (q x+\phi)}{A}\left(R+x-\frac{z y q}{A}+\frac{u}{A}-\frac{y q w}{A}\right), \\
V_{2}=\mathbf{V}_{T} \cdot \tilde{\mathbf{a}}_{2}=\dot{v}-\Omega \sin (q x+\phi)\left(R+x-\frac{z y q}{A}+\frac{u}{A}-\frac{y q w}{A}\right), \\
V_{3}=\mathbf{V}_{T} \cdot \tilde{\mathbf{a}}_{3}=\dot{w}-\frac{y q V_{x}}{A}-\frac{\Omega \sin (q x+\phi)}{A}\left(R+x-\frac{z y q}{A}+\frac{u}{A}-\frac{y q w}{A}\right),
\end{array}\right.
$$

where a super dot implies the first-order derivation of time. Then the kinetic energy of the cantilever twisted plate yields

$$
\begin{aligned}
& T= \iint_{V} \frac{1}{2} \rho V_{i}^{2} d V \\
&=\iiint_{v} \frac{1}{2} \rho\left(\dot{u}^{2}+\dot{v}^{2}+\dot{w}^{2}+\Omega^{2}\left(y \sin (q x+\varphi)+\frac{z \cos (q x+\varphi)}{A}+\frac{u y q \cos (q x+\varphi)}{A}\right.\right. \\
&\left.+v \sin (q x+\varphi)+\frac{w \cos (q x+\varphi)}{A}\right)^{2}+\Omega^{2}\left(R+x-\frac{z y q}{A}+\frac{u}{A}-\frac{y q w}{A}\right)^{2}+\frac{2 \Omega \dot{u}}{A}(y \sin (q x+\varphi) \\
&\left.+\frac{z \cos (q x+\varphi)}{A}\right)-\frac{2 \Omega y q \cos (q x+\varphi) \dot{u}}{A}\left(R+x-\frac{z y q}{A}\right)-2 \Omega \sin (q x+\varphi) \dot{v}\left(R+x-\frac{z y q}{A}\right) \\
&-\frac{2 \Omega y q \dot{w}}{A}\left(y \sin (q x+\varphi)+\frac{z \cos (q x+\varphi)}{A}\right)-\frac{2 \Omega \cos (q x+\varphi) \dot{w}}{A}\left(R+x-\frac{z y q}{A}\right) \\
&+\Omega\left(\frac{2 \dot{u} v}{A} \sin (q x+\varphi)-\frac{2 u \dot{v}}{A} \sin (q x+\varphi)+2 \dot{u} w \cos (q x+\varphi)-2 u \dot{w} \cos (q x+\varphi)\right. \\
&\left.\left.+\frac{2 y q}{A} \sin (q x+\varphi) w \dot{v}-\frac{2 y q}{A} \sin (q x+\varphi) \dot{w} v\right)\right) d V .
\end{aligned}
$$

The centrifugal force along $x, y$ and $z$ axes can be expressed as:

$$
\begin{aligned}
& N_{c 1}=\int_{x}^{a} \mathbf{F}_{c} \cdot \tilde{\mathbf{a}}_{1} A d x, \\
& N_{c 2}=\int_{y}^{ \pm b / 2} \mathbf{F}_{c} \cdot \tilde{\mathbf{a}}_{2} d y, \\
& N_{c 3}=\mathbf{F}_{c} \cdot \tilde{\mathbf{a}}_{3},
\end{aligned}
$$


where $\mathbf{F}_{c}$ is given as

$$
\mathbf{F}_{c}=-\boldsymbol{\Omega} \times(\boldsymbol{\Omega} \times \mathbf{r})=\left\{\begin{array}{l}
\mathbf{F}_{c x}=\rho \Omega^{2}\left(R+x+\frac{z y k}{A}\right) \tilde{\mathbf{i}}, \\
\mathbf{F}_{c y}=\rho \Omega^{2}\left(y \sin (q x+\varphi)+\frac{z}{A} \cos (q x+\varphi)\right) \sin (\varphi) \tilde{\mathbf{j}}, \\
\mathbf{F}_{c z}=\rho \Omega^{2}\left(y \sin (q x+\varphi)+\frac{z}{A} \cos (q x+\varphi)\right) \cos (\varphi) \tilde{\mathbf{k}},
\end{array}\right.
$$

where $\rho$ is the density of the twisted plate.

The potential energy resulting from centrifugal force is given by

$$
U_{p}=\iiint_{V}\left(N_{c 1} u_{1}+N_{c 2} u_{2}+N_{c 3} u_{3}\right) d V,
$$

where $u_{i},(i=1,2,3)$ are the displacements along centrifugal force and they can take the form, see $[26,28]$

$$
\begin{aligned}
& u_{1}=u_{0}+\frac{1}{2}\left(\left(\frac{\partial w_{0}}{\partial x}\right)^{2}+\left(\frac{\partial v_{0}}{\partial x}\right)^{2}\right), \\
& u_{2}=v_{0}+\frac{1}{2}\left(\left(\frac{\partial w_{0}}{\partial y}\right)^{2}+\left(\frac{\partial u_{0}}{\partial y}\right)^{2}\right), \\
& u_{3}=w_{0} .
\end{aligned}
$$

The work done by the external transverse force is given by

$$
W_{F}=\iint_{s} p w d s
$$

\subsection{Time-dependent dynamic loading}

Now suppose that the cantilever twisted plate is exposed to two kinds of uniform dynamic loads. They are step loading and air-blast loading, respectively. The step loading is represented by

$$
p(t)= \begin{cases}p_{0}, & 0<t<r t_{p}, \\ 0, & t<0, \quad t>r t_{p},\end{cases}
$$

where $p_{0}$ denotes the peak pressure, $r$ is shock pulse length factor, and $t_{p}$ indicates positive phase duration.

Exponential air blast loading considered here is written as, see [39]

$$
p(t)=p_{0}\left(1-t / t_{p}\right) e^{-\frac{\alpha t}{t_{p}}},
$$

where $t$ is the time and $\alpha$ denotes the waveform decay parameter. It includes the Friedlander exponential decay function. 


\subsection{Mode shapes}

To find the mode shapes of the cantilever twisted shell, the Rayleigh-Ritz method is employed in this section and a linear free vibration system can be obtained by ignoring the work done by the external force and nonlinear terms. The free vibration can be expressed in following form

$$
\begin{aligned}
& u_{0}(x, y, t)=\tilde{U}(x, y) e^{i \omega t}, \\
& v_{0}(x, y, t)=\tilde{V}(x, y) e^{i \omega t}, \\
& w_{0}(x, y, t)=\tilde{W}(x, y) e^{i \omega t} .
\end{aligned}
$$

The non-dimensional algebraic polynomial functions will be applied since there are many advantages of algebraic polynomial, see [40]. Thus, the displacement functions $\tilde{U}, \tilde{V}$ and $\tilde{W}$ are given as

$$
\begin{aligned}
& \tilde{U}(\xi, \eta)=\sum_{i=1}^{I} \sum_{j=1}^{J} A_{i j} \xi^{i} \eta^{j-1}, \\
& \tilde{V}(\xi, \eta)=\sum_{k=1}^{K} \sum_{l=1}^{L} B_{k l} \xi^{k} \eta^{l-1}, \\
& \tilde{W}(\xi, \eta)=\sum_{m=1}^{M} \sum_{n=1}^{N} C_{m n} \xi^{m+1} \eta^{n-1},
\end{aligned}
$$

where $\xi=\frac{x}{a}, \eta=\frac{2 y}{b}, A_{i j}, B_{k l}$ and $C_{m n}$ are coefficients that will to be determined. The $(I, J),(K, L)$ and $(M, N)$ are the number of the approximate solution for each displacement component and they should assure convergence of the approximate solution.

Substituting the Eqs. (2.27), (2.28) into Eqs. (2.11), (2.19) and Eq. (2.22), respectively, then using the Rayleigh-Ritz method lead to

$$
\begin{array}{ll}
\frac{\partial\left(T_{\max }-U_{\max }-U_{p \max }\right)}{\partial A_{i j}}=0, & (i=1, \cdots, I ; \quad j=1, \cdots, J), \\
\frac{\partial\left(T_{\max }-U_{\max }-U_{p \max }\right)}{\partial B_{k l}}=0, & (k=1, \cdots, K ; \quad l=1, \cdots, L), \\
\frac{\partial\left(T_{\max }-U_{\max }-U_{p \max }\right)}{\partial C_{m n}}=0, & (m=1, \cdots, M ; \quad n=1, \cdots, N) .
\end{array}
$$

With the aid of Eq. (2.29), we can have the algebraic equation as follows

$$
\left(K-\omega^{2} M\right) X=0,
$$

where vector

$$
\mathbf{X}=\left[\tilde{U}_{11} \cdots \tilde{U}_{I J}, \tilde{V}_{11} \cdots \tilde{V}_{K L}, \tilde{W}_{11} \cdots \tilde{W}_{M N}\right]^{T},
$$


Table 1: Comparisons of the first four frequencies of cantilever twisted laminate plate with $\mathrm{Hu}$ and Lim [41] $\left(\omega_{0}=\omega b \sqrt{\rho / E}\right)$.

\begin{tabular}{||cccccc||}
\hline Twisted angle & Mode No. & 1 & 2 & 3 & 4 \\
\hline \multirow{4}{*}{$10^{\circ}$} & Present & 0.00083449 & 0.0051764 & 0.0084883 & 0.014642 \\
& Hu and Lim & 0.00085688 & 0.0052237 & 0.0086112 & 0.014962 \\
& Error & $-2.68 \%$ & $-0.91 \%$ & $-1.45 \%$ & $-2.19 \%$ \\
\hline \multirow{2}{*}{$20^{\circ}$} & Present & 0.00082396 & 0.0050470 & 0.013530 & 0.014567 \\
& Hu and Lim & 0.00084039 & 0.0051040 & 0.013235 & 0.014906 \\
& Error & $-1.99 \%$ & $-1.13 \%$ & $2.18 \%$ & $-2.33 \%$ \\
\hline
\end{tabular}

Table 2: Comparison of the first four non-dimensional frequencies obtained from [20] and present.

\begin{tabular}{|c|c|c|c|c|c|}
\hline Lay-up & Solution method & 1 & 2 & 3 & 4 \\
\hline \multirow{7}{*}{$30^{\circ}$} & $5 * 5 * 3$ & 1.637 & 2.092 & 3.817 & 5.1599 \\
\hline & error & $1.03 \%$ & $0.65 \%$ & $2.76 \%$ & $2.10 \%$ \\
\hline & $6^{*} 6 * 3$ & 1.6226 & 2.0903 & 3.7676 & 5.1266 \\
\hline & error & $0.15 \%$ & $0.56 \%$ & $1.49 \%$ & $1.46 \%$ \\
\hline & $7 * 7 * 3$ & 1.6167 & 2.0781 & 3.7106 & 5.0552 \\
\hline & error & $-0.22 \%$ & $-0.02 \%$ & $-0.02 \%$ & $0.07 \%$ \\
\hline & Qatu & 1.6202 & 2.0785 & 3.7115 & 5.0517 \\
\hline \multirow{7}{*}{$45^{\circ}$} & $5 * 5 * 3$ & 1.7092 & 2.2538 & 3.934 & 4.764 \\
\hline & error & $1.29 \%$ & $0.68 \%$ & $2.94 \%$ & $1.88 \%$ \\
\hline & $6 * 6 * 3$ & 1.6919 & 2.2524 & 3.8806 & 4.7589 \\
\hline & error & $0.28 \%$ & $0.62 \%$ & $1.61 \%$ & $1.77 \%$ \\
\hline & $7 * 7 * 3$ & 1.6865 & 2.2401 & 3.8232 & 4.6828 \\
\hline & error & $-0.04 \%$ & $0.07 \%$ & $0.13 \%$ & $0.18 \%$ \\
\hline & Qatu & 1.6871 & 2.2385 & 3.8182 & 4.6745 \\
\hline
\end{tabular}

$\mathbf{K}$ and $\mathbf{M}$ are stiffness and mass matrix, respectively. It is known that when the determinant of coefficient is zero we can have nontrivial vector $\mathbf{X}$. Then the mode shape and natural frequency will be calculated.

To validate mode shapes obtained present, the first four dimensionless frequencies $\left(\omega_{0}=\omega b \sqrt{\rho / E}\right)$ of the twisted laminate $\left[45^{\circ} /-45^{\circ} /-45^{\circ} / 45^{\circ}\right]$ plates with $E_{11}=60.7 \mathrm{GPa}$, $E_{22}=24.8 \mathrm{GPa}, G_{12}=12.0 \mathrm{GPa}, \rho=2700 \mathrm{~kg} / \mathrm{m}^{3}, \mu=0.23, \frac{a}{b}=3, \frac{h}{b}=0.01$ and $\theta=10^{\circ} / 20^{\circ}$ are compared with those obtained by $\mathrm{Hu}$ and Lim [41], which is listed in Table 1. It is clearly from Table 1 that a good agreement is shown with the maximum error $-2.68 \%$.

In Table 2 and Fig. 2, comparison of the first four dimensionless frequencies and mode shapes obtained from Qatu and leissa [20] and present are shown. The comparison shows that the maximum error is less than $2.76 \%$ when trial function are $5 \times 5 \times 3,6 \times 6 \times 3,7 \times$ $7 \times 3$, which prove that the eigenvalues are convergence and acceptable in present. The present mode shapes using trial function of $7 \times 7 \times 3$ are excellent agreement with the [20]. The material and geometric of the plate are Angle-ply plate $\left(30^{\circ} /-30^{\circ} / 30^{\circ}\right)$ with $a=b=1$, $b / h=100, E_{11}=138 \mathrm{GPa}, E_{22}=8.69 \mathrm{GPa}, G_{12}=7.10 \mathrm{GPa}, \rho=1600 \mathrm{~kg} / \mathrm{m}^{3}, \omega=\omega_{0} \frac{a^{2}}{h} \sqrt{\frac{\rho}{E}}$ and 

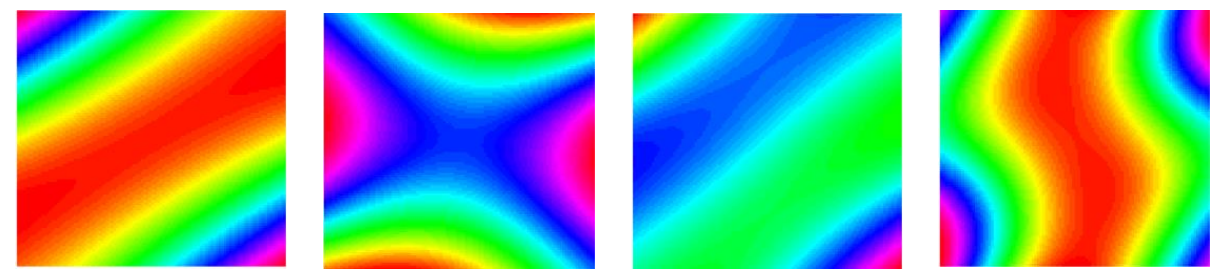

(a) $30^{\circ}$ present $7^{*} 7^{*} 3$
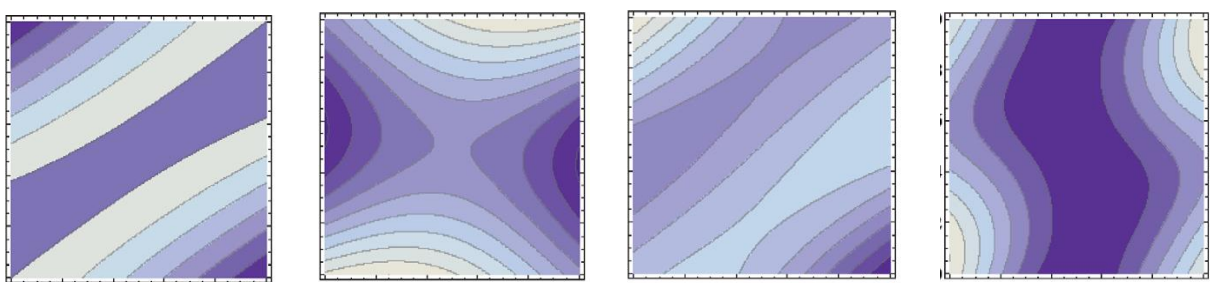

(b) Ref [20]
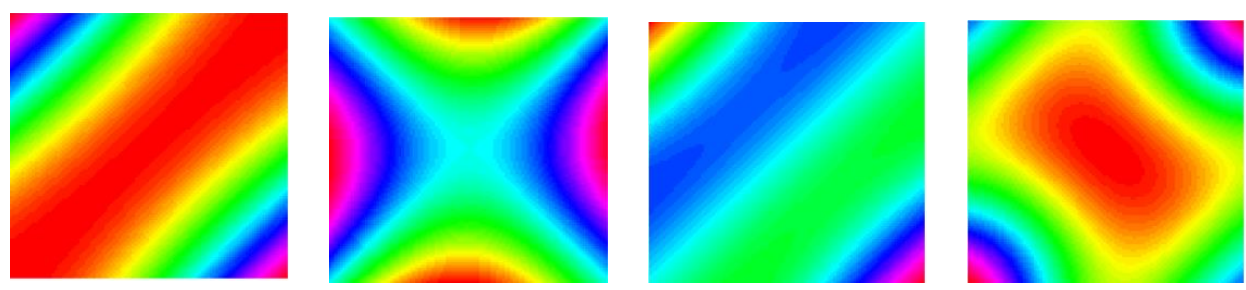

(c) $45^{\circ}$ present $7^{*} 7^{*} 3$
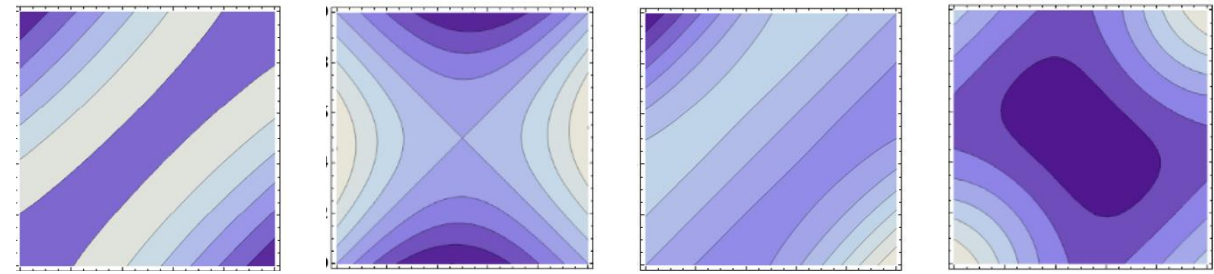

(d) Ref [20]

Figure 2: Comparison of the first four mode shapes obtained from [20] and present.

$\mu=0.3$.

\subsection{Nonlinear dynamic equations}

The first four frequencies and mode shapes of the system used in this article are given in Table 3 and Fig. 3, respectively. 


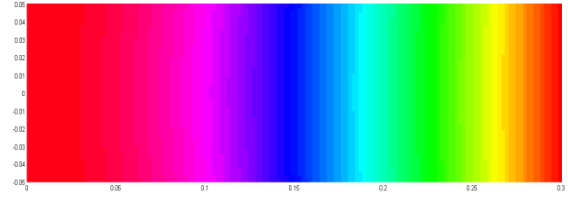

(a) 1st mode shape

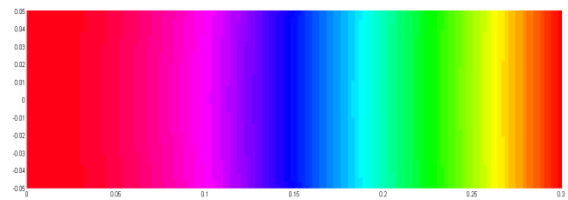

(c) 3rd mode

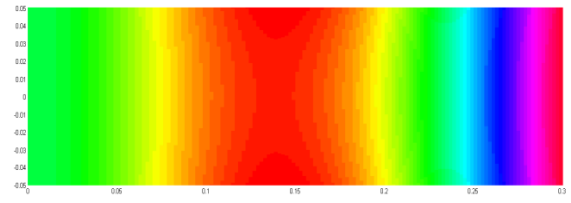

(b) 2nd mode shape

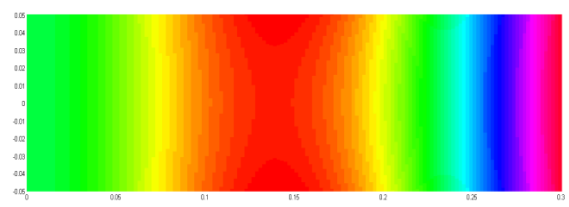

(d) 4th mode

Figure 3: The first four mode shapes of cantilever twisted plate with $a / b=3$.

Table 3: The first four frequencies of cantilever twisted plate.

\begin{tabular}{||c|cccc||}
\hline \multirow{2}{*}{ Aspect ratios } & \multicolumn{4}{|c||}{ Frequencies $(\mathrm{Hz})$} \\
\cline { 2 - 5 } & 1 & 2 & 3 & 4 \\
\hline $3(7 \times 7 \times 3)$ & 26.3809 & 159.7723 & 256.6637 & 468.0655 \\
\hline
\end{tabular}

Based on the mode shapes obtained above, the expansions of the middle surface displacements can be given by

$$
\begin{aligned}
& u_{0}(\xi, \eta, t)=\sum_{s=1}^{S} u_{s}(t) \tilde{U}^{s}(\xi, \eta), \\
& v_{0}(\xi, \eta, t)=\sum_{s=1}^{S} v_{s}(t) \tilde{V}^{s}(\xi, \eta), \\
& w_{0}(\xi, \eta, t)=\sum_{s=1}^{S} v_{s}(t) \tilde{W}^{s}(\xi, \eta),
\end{aligned}
$$

where $t$ is the time, $u_{s}, v_{s}$ and $w_{s}$ are time dependent, $\xi$ and $\eta$ have the same means with Eq. (2.28). And $\tilde{U}^{s}, \tilde{V}^{s}$ and $\tilde{W}^{s}$ are the eigenvectors corresponding to the sth natural frequency.

In order to get the nonlinear ordinary differential equations of motion Lagrange function is defined as

$$
L=T-U-U_{p}+W_{F}
$$

According to the Lagrange formulation, dynamic equations of the cantilever twisted shell can be written as:

$$
\frac{d}{d t}\left(\frac{\partial L}{\partial \dot{\mathbf{q}}_{r}}\right)-\frac{\partial L}{\partial \mathbf{q}_{r}}=0,
$$


where $\mathbf{q}_{r}=\left[u_{1}(t) \cdots u_{s}(t), v_{1}(t) \cdots v_{s}(t), w_{1}(t) \cdots w_{s}(t)\right]^{T}$.

Because the transverse displacement is mainly considered here, therefore we neglect all of the in-plane inertia terms in Eq. (2.33) and in-plane displacements are expressed in terms of transverse displacement by solving the algebraic equations, see $[42,43]$. We mainly take transverse nonlinear dynamic response of the cantilever twisted plate in the first four modes into account. Consequently, the nonlinear dynamic equations of motion of the cantilever twisted plate described by retaining four transverse modes are obtained as follows

$$
\hat{\mathbf{M}} \ddot{\mathbf{w}}+\hat{\mathbf{K}} \mathbf{w}+\mathbf{N}_{2}(\mathbf{w})+\mathbf{N}_{3}(\mathbf{w})=\zeta p_{e}+\mathbf{f}_{n},
$$

where $\hat{\mathbf{M}}$ and $\hat{\mathbf{K}}$ are the mass and linear stiffness matrix respectively, $\mathbf{N}_{2}(\mathbf{w})$ is quadratic nonlinear component, $\mathbf{N}_{3}(\mathbf{w})$ gives cubic nonlinear terms. It is very interesting that there are different linear and nonlinear stiffness with the changes of the geometric and rotation speed of the plate. Vectors $\mathbf{p}_{e}$ and $\mathbf{f}_{n}$ are time-dependent dynamic load and centrifugal force respectively. $\zeta$ is a coefficient which introduced in calculating the work done by the external transverse force.

\section{Nonlinear dynamic response}

To analyze the nonlinear dynamic response of the cantilever twisted plate, the first four mode shapes are selected and the double-precision variable-coefficient ordinary differential equation solver (DVODE) [44-47] is used with the aid of FORTRAN.

\subsubsection{Validation and convergence}

To validate present formulation, response results and compiled procedure, the transient dynamic response curves of the plate which is loaded by step loading and air-blast loading, respectively, are plotted in Fig. 4 and compared with [48,49].

Comparison 1: The validation has been done firstly by comparing the central displacement for a simply supported homogeneous isotropic aluminum plate $(a=b=0.2 \mathrm{~m}, L=$ $0.01 \mathrm{~m}$ ) subjected to uniform step loading between present results and those given by Reddy [48] in Fig. 4(a). The intensity of the step loading is $-10^{6} \mathrm{~N} / \mathrm{m}^{2}$. It can be seen that there is excellent agreement with those calculated by Reddy.

Comparison 2: In this comparison, a composite laminated square plate $\left(0^{\circ} / 90^{\circ} / 0^{\circ}\right)$ under blast pressure $\left(p_{\max }=3447 \mathrm{kPa}\right)$ with length $2.54 \mathrm{~m}$ and highness $0.17 \mathrm{~m}$ is selected. The material properties are $E_{1}=132.4 \mathrm{GPa}, E_{2}=10.8 \mathrm{GPa}, G_{12}=5.6 \mathrm{GPa}, \rho=1443 \mathrm{~kg} / \mathrm{m}^{3}$ and $v_{12}=0.24$, respectively. Parameters about blast loading are $\alpha=2.0, r=1.0$ and $t_{p}=0.1 \mathrm{~s}$, respectively. The results obtained by present method and Kazanc1 [49] is shown in Fig. 4(b) and a good agreement can be observed.

Fig. 5 points out the convergence of the cantilever twisted plate subjected to step and air blast loading, respectively. It is clearly that, in the case of zero rotation speed, the 


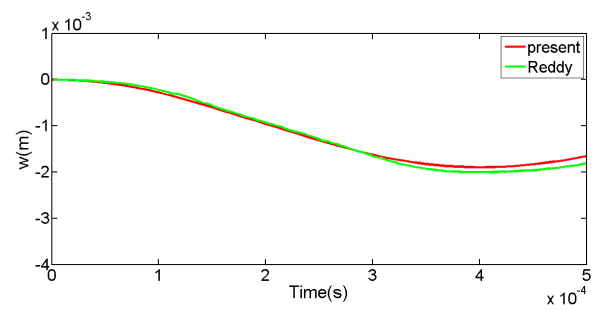

(a)

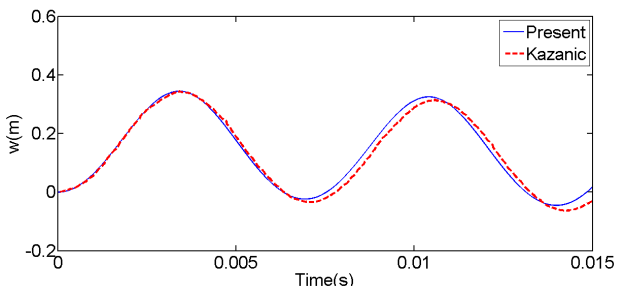

(b)

Figure 4: (a) Comparison of transient response by center deflection of plate subjected to step loading; (b) Comparison of transient response by center deflection of plate subjected to blast loading.

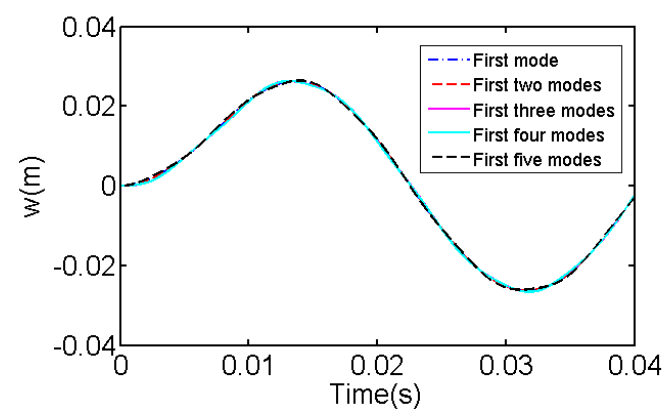

(a) under air-blast loading $(\Omega=0)$

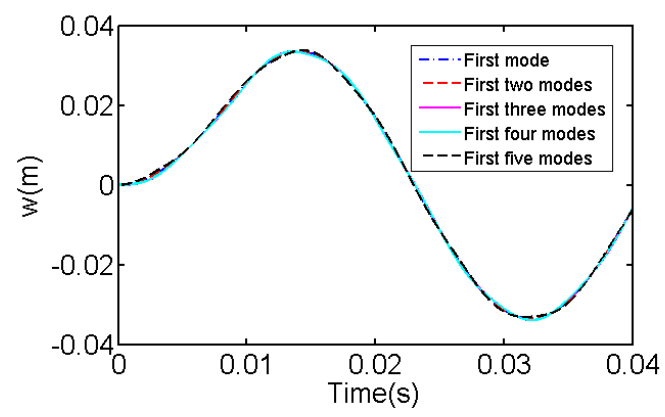

(c) under step loading $(\Omega=0)$

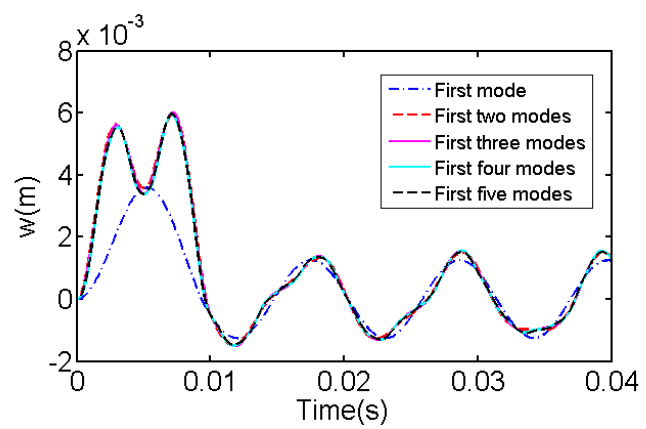

(b) under air-blast loading $(\Omega=4000 \mathrm{rpm})$

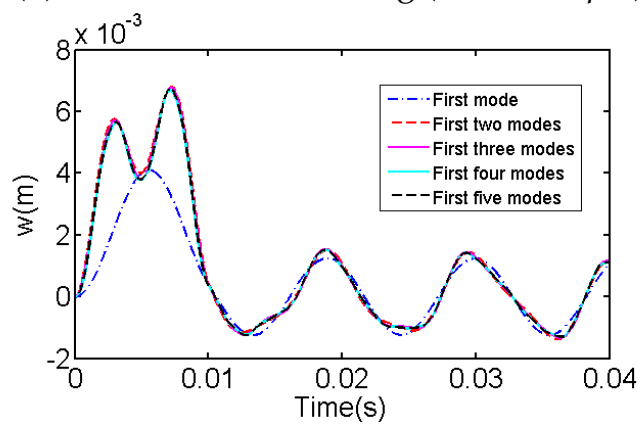

(d) under step loading $(\Omega=4000 \mathrm{rpm})$

Figure 5: Convergence of the corner point deflection of cantilever twisted plate in free edge.

corner point deflection of cantilever twisted plate in free edge is nearly the same when the first five modes are used respectively. But if the rotating cantilever twisted plate is studied at least first two modes should be included based on the results plotted in Figs. 5(c) and (d). In present study first four modes is enough to be used to analyze the dynamic response of this system. 


\subsection{Transient response}

The transient nonlinear dynamic responses of the cantilever twisted plate subjected to time-dependent loading are investigated numerically. Unless stated otherwise, the material, geometrical and loading parameters considered in the analysis are given as $E=$ $2 \times 10^{11} \mathrm{GPa}, \rho=7850 \mathrm{~kg} / \mathrm{m}^{3}, \mu=0.3, p_{0}=10^{4} \mathrm{~Pa} . \Omega=4000 \mathrm{rpm}, t_{p}=0.01 \mathrm{~s}, a=300 \mathrm{~mm}$, $b=100 \mathrm{~mm}, \theta=30^{\circ}, h=3 \mathrm{~mm}, \varphi=45^{\circ}$ and $R=200 \mathrm{~mm}$, respectively. The shock pulse length factor is $r=1.0$, The time process diagram of central displacements of the cantilever twisted plate are used to demonstrate the transient responses of the cantilever twisted plate.

Fig. 6 depicts the effect of the stagger angle $\varphi$ on time process diagram of central point deflection of the plate and exposed to the uniform dynamic step loading and airblast loading. The geometric parameters are: $a=300 \mathrm{~mm}, b=100 \mathrm{~mm}, \theta=30^{\circ}, h=3 \mathrm{~mm}$, $\varphi=0^{\circ} / 45^{\circ} / 90^{\circ}, R=200 \mathrm{~mm}$, respectively. Figs. 6(a) and (b) represents the transient nonlinear dynamics of the center point of the plate under air-blast loading and step loading, respectively.

It can be seen from Figs. 6(a) and (b) that in forced motion regimes the largest central deflections are detected for stagger angle $\varphi=0^{\circ}$. But in free motion regimes the largest central deflections are found for the plate with stagger angle $\varphi=45^{\circ}$. Both in free and forced motion regions, one can obtain a lower central deflections for the plate with $\varphi=90^{\circ}$. It is clearly that the frequencies of the nonlinear dynamic response of the plate exposed to step loading are larger than that of the plate subjected to air-blast loading. It also appears that stagger angle can affect not only the deflection of the plate but also the shapes of them.

The effects of the length-thickness ratio, when parameters $a=300 \mathrm{~mm}, b=100 \mathrm{~mm}$, $\theta=30^{\circ}, \varphi=45^{\circ}$ and $R=200 \mathrm{~mm}, a=300 \mathrm{~mm}$ are fixed, on time-history of central deflection of the twisted plate acted by the uniform time-dependent loading are plotted in Figs. 7(a) and (b).

As expected, in these two cases which the plate is acted by air-blast loading and step loading respectively, central displacement amplitude of the twisted plate increases with

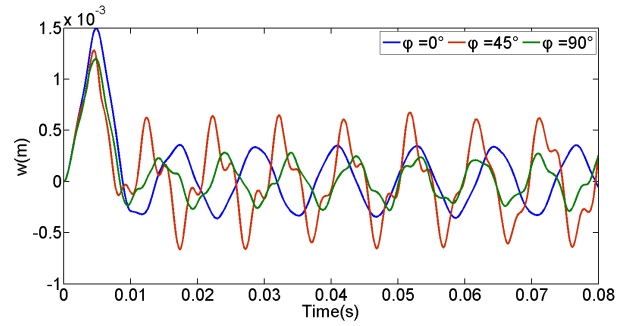

(a) under air-blast loading

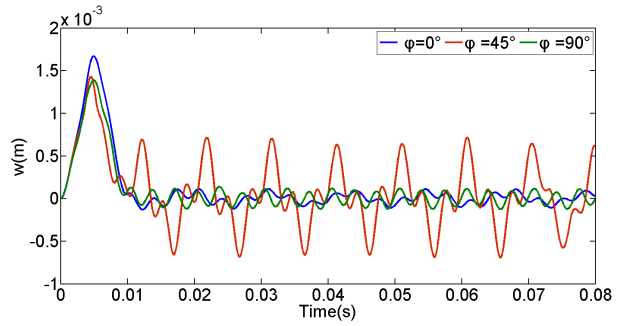

(b) under step loading

Figure 6: Center displacements of the cantilever twisted plate with different stagger angles under air-blast loading and step loading. 


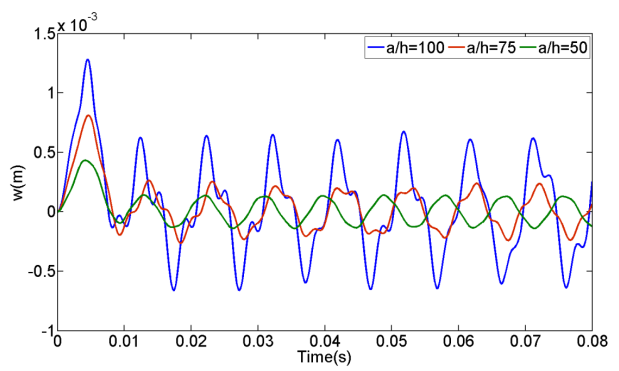

(a) under air-blast loading

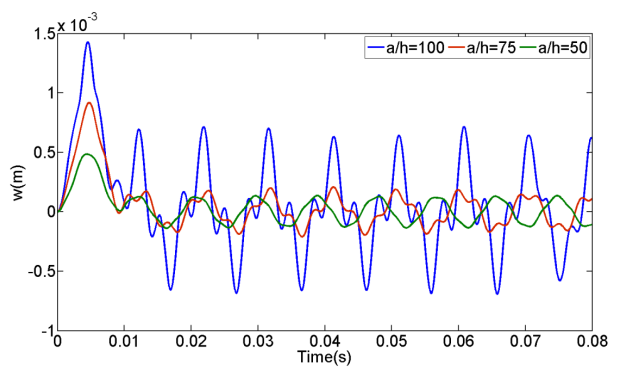

(b) under step loading

Figure 7: Central displacements of the cantilever twisted plate with different length-thickness ratio under airblast loading and step loading.

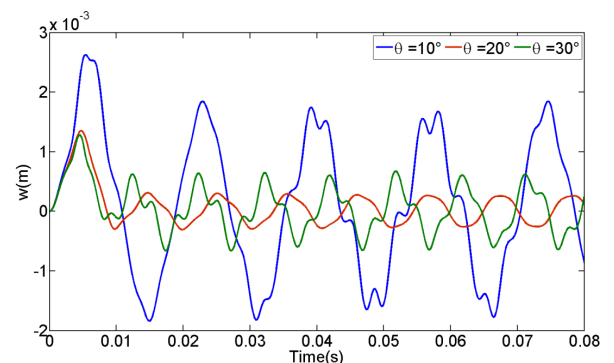

(a) under air-blast loading

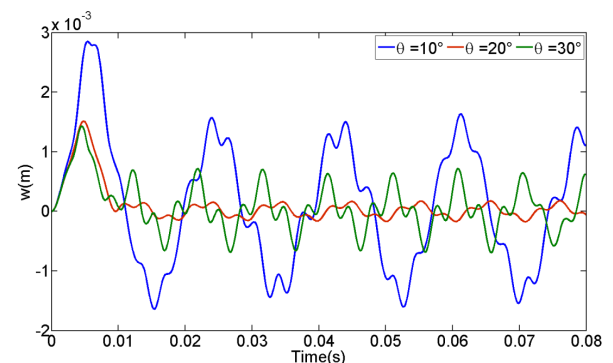

(b) under step loading

Figure 8: Central displacements of the cantilever twisted plate with different twisted angles under air-blast loading and step loading.

the decrease in length-thickness ratio either in forced motion region or in free motion region. The frequencies and transverse deflection of the cantilever twisted plate have an opposite variation tendency. These response results are due to the stiffness of the twisted plate decreases as the thickness becoming thinner. And it also be seen that the difference of the central deflection between $a / h=100$ and $a / h=75$ is far greater than that between $a / h=75$ and $a / h=50$. It illustrates that as a nonlinear system the central deflection does not increase linearly with the change of thickness.

Fig. 8 reveals the effect of twisted angle on time-history of central deflection of the twisted plate $\left(a=300 \mathrm{~mm}, b=100 \mathrm{~mm}, \theta=10^{\circ} / 20^{\circ} / 30^{\circ}, h=3 \mathrm{~mm}, \varphi=45^{\circ}\right)$. It is worth noting that in forced motion region the central deflection decreases with the increase of twisted angle of the plate. And the amplitude for $\theta=10^{\circ}$ is almost twice as much as that of $\theta=20^{\circ}$ and $\theta=30^{\circ}$. What's more interesting, in free motion region the deflection of the nonlinear dynamics response for $\theta=10^{\circ}$ and $\theta=30^{\circ}$ is larger than that for $\theta=20^{\circ}$. Compared Figs. 8(a) and (b), it can be noted that the amplitude of the response of air-blast loading is a little higher than that of step loading for $\theta=10^{\circ}$ and $\theta=20^{\circ}$ in free motion region. In forced region the step loading can make the plate cause higher response than step loading. Additionally, the different shapes of the deflection curve are shown for 


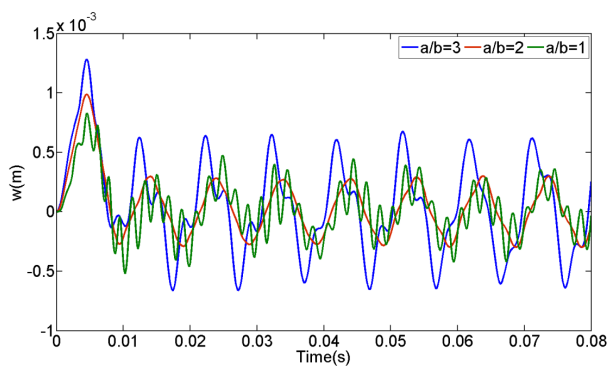

(a) under air-blast loading

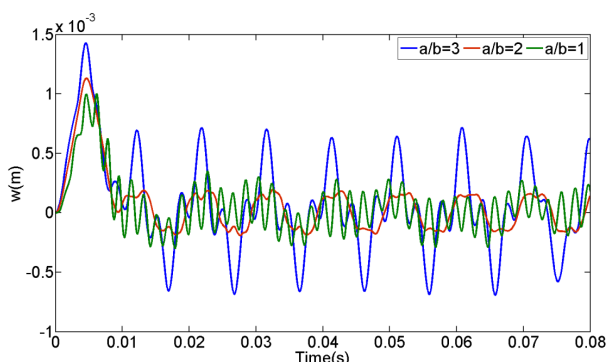

(b) under step loading

Figure 9: Central displacements of the cantilever twisted plate with different aspect ratios under air-blast loading and step loading.

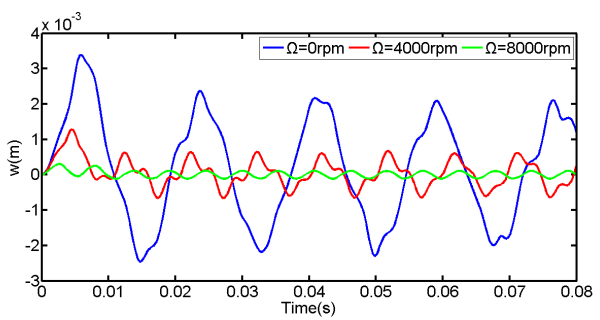

(a) under air-blast loading

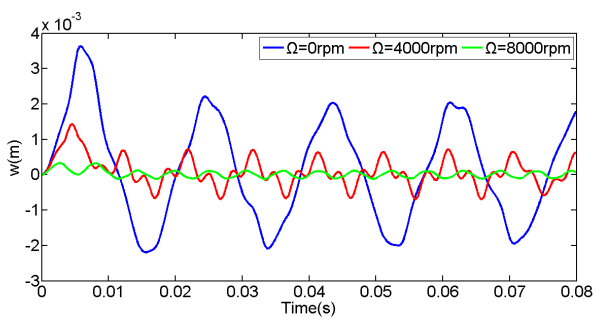

(b) under step loading

Figure 10: Central displacements of the cantilever twisted plate with different rotation speed under air-blast loading and step loading.

different twisted angles.

The nonlinear transient responses of the cantilever twisted plated for the cantilever twisted plate $\left(a=300 \mathrm{~mm}, \theta=30^{\circ}, h=3 \mathrm{~mm}, \varphi=45^{\circ}, b=100 \mathrm{~mm} / 200 \mathrm{~mm} / 300 \mathrm{~mm}\right)$ under air-blast loading are researched in Fig. 9(a). The results of the plate under step loading are given for direct comparison in Fig. 9(b). Obviously, the shapes of the nonlinear transient dynamic response curve are different. The dynamic central deflection is maximum for the twisted plate with aspect ratio is 3 . It is interesting that the minimum of central deflection is the plate with aspect ratio is 2 but not that with $a / b=1$. The reason is that in this dynamic system the nonlinearity is considered. For the plate subjected to step loading, the response amplitude of it in forced motion region is slightly smaller than that subjected to air-blast loading. But in free motion region the opposite case is true.

The influence of rotation speed on transient response of the twisted plate $(a=300 \mathrm{~mm}$, $b=100 \mathrm{~mm}, \theta=30^{\circ}, h=3 \mathrm{~mm}, \varphi=45^{\circ}$ and $\Omega=0 / 4000 / 8000 \mathrm{rpm}$ ) can be studied intuitively by time process diagram of plate central deflection shown in Fig. 10. One can see that the transient dynamic response of the plate under air-blasting loading and step loading is in a similar manner. The amplitude is always quite small both in forced motion region and free motion region as time grows in case $\Omega=8000 \mathrm{rpm}$. It is clear that the rotation speed can be considered as an important factor that make the system stiffening. The shape 


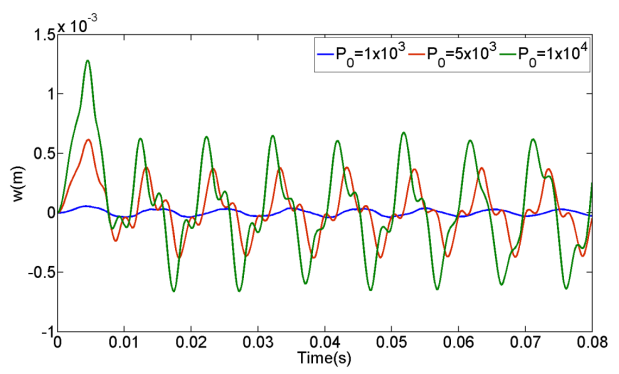

(a) under air-blast loading

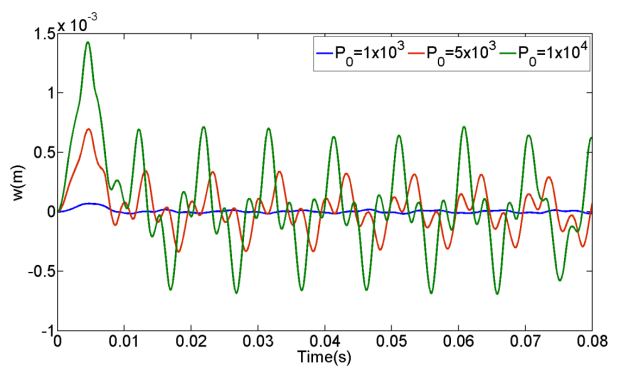

(b) under step loading

Figure 11: Central displacements of the cantilever twisted plate with different load intensity under air-blast loading and step loading.

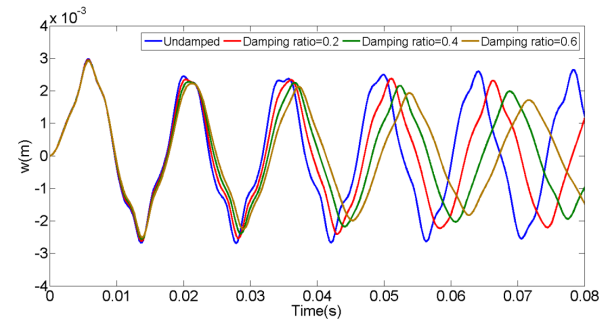

(a) under air-blast loading

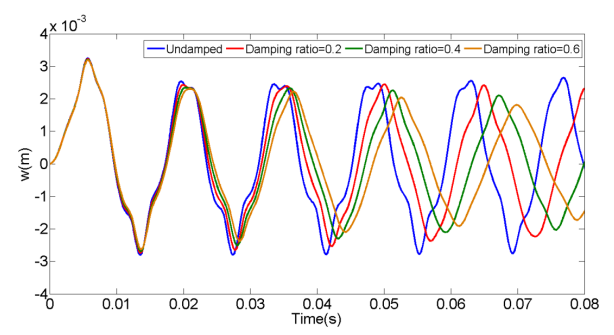

(b) under step loading

Figure 12: Comparison of central displacements damped and undamped plates under air-blast loading and step loading (rotation speed $=0$ ).

responses are in same way for two kinds of loading.

Fig. 11 displays the effect of the load amplitude on the transient dynamic response of the cantilever twisted plate $\left(a=300 \mathrm{~mm}, b=100 \mathrm{~mm}, \theta=30^{\circ}, h=3 \mathrm{~mm}, \varphi=45^{\circ}\right)$. It illustrates that both loading show nearly the same deflection amplitudes for the plate. Under the air-blast loading and step loading, the response frequencies of plate are nearly same too. Furthermore, it indicates that the first four modes are enough for us to analyze the nonlinear transient dynamic response of the system because the response frequencies are in the region of the first four natural frequencies.

In order to consider the effect of transverse viscous damping on nonlinear transient response of the system the viscous damping force are added in Eqs. (2.24) and (2.34) can be rewritten as

$$
\begin{aligned}
& W_{F}=\iint_{s} p w d s-\iint_{s} \tau \dot{w} w d s, \\
& \hat{\mathbf{M}} \ddot{\mathbf{w}}+\hat{\mathbf{C}} \dot{\mathbf{w}}+\hat{\mathbf{K}} \mathbf{w}+\mathbf{N}_{2}(\mathbf{w})+\mathbf{N}_{3}(\mathbf{w})=\zeta p_{e}+\mathbf{f}_{n},
\end{aligned}
$$

where $\hat{\mathbf{C}}$ is the viscous damping matrix. Following the study by Alijani and Amabili [50], the same damping ratio is applied to do numerical simulation. Figs. 12-14 shows the non- 


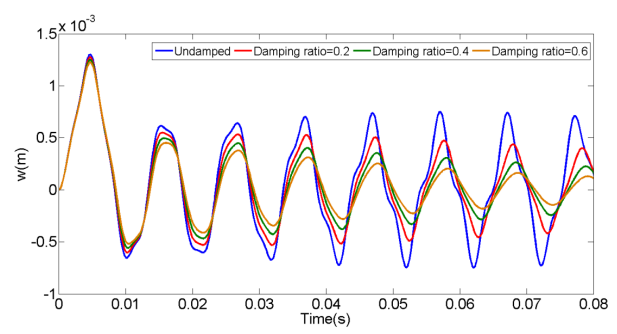

(a) under air-blast loading

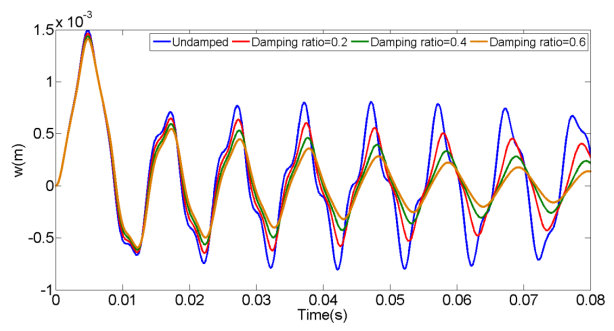

(b) under step loading

Figure 13: Comparison of central displacements damped and undamped plates under air-blast loading and step loading (rotation speed $=4000 \mathrm{rpm})$.

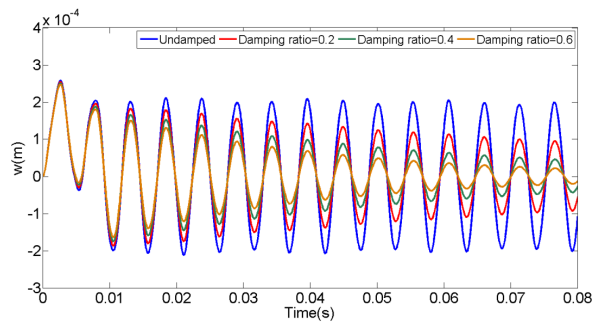

(a) under air-blast loading

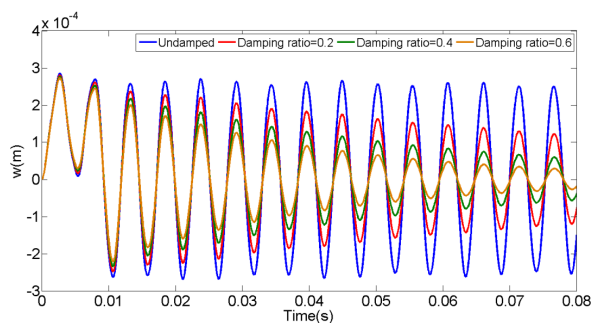

(b) under step loading

Figure 14: Comparison of central displacements damped and undamped plates under air-blast loading and step loading (rotation speed $=8000 \mathrm{rpm})$.

linear dynamics response of the undamped cantilever twisted late and those with viscous damping ratio taking the value of $0.2,0.4$ and 0.6 when the rotation are $0 \mathrm{rpm}, 4000 \mathrm{rpm}$ and $8000 \mathrm{rpm}$, respectively. One can see that with the increasing of the rotation speed the plate will be damped more quickly after the blast loading, the vibration frequencies of it will be increased but the amplitude decrease greatly under the same damp.

\section{Conclusions}

The transient nonlinear dynamic responses of the cantilever twisted rectangular plate under the air-blast loading and step loading respectively are investigated in detail. Although the homogeneous and isotropic material is applied in this study, it might be helpful for composite twisted plates. The use of variable mode functions by polynomial functions according to the twist angles and geometric of the plate makes it more accurate to describe the dynamic system than that using the classic cantilever beam functions and the free-free beam functions. The comparison researches are carried out between the results from ANSYS and presents. By using Lagrange equations and nonlinear geometric theory, nonlinear dynamic equations of motion of the cantilever twisted plate are derived. The equations of motion describing the transient nonlinear dynamic response are 
reduced to a four degree of freedom dynamic system which expressed by out-plane displacement for the first time. The effects of twisted angle, stagger angle, rotation speed, load intensity and other plate parameters have been investigated. It is observed that the amplitude of the central deflection of the twisted plate is very close when it is exposed to step loading in comparison to air blast loadings. It is also observed that the effect of twisted angle, stagger angle and length-width ratio on the transient response of the plate are more complex. Numerical results indicate that the first four modes are enough for us to analyze the nonlinear transient dynamic response of the system. It is indicated that the first four modes are enough for us to analyze the nonlinear transient dynamic response of the system because the response frequencies are in the region of the first four natural frequencies. Also, it can be concluded that with the increasing of the rotation speed the plate will be damped more quickly after the blast loading, the vibration frequencies of it will be increased but the amplitude decrease greatly under the same damp.

\section{Acknowledgements}

The authors acknowledge the financial support of National Natural Science Foundation of China through grant Nos. 11872127, 11832002 and 11732005, Fundamental Research Program of Shenzhen Municipality No. JCYJ20160608153749600 and the Project of Highlevel Innovative Team Building Plan for Beijing Municipal Colleges and Universities No. IDHT20180513 and the project of Qin Xin Talents Cultivation Program, Beijing Information Science \& Technology University QXTCP A201901.

\section{References}

[1] G. HAN, Y. S. CHEN AND X. D. WANG, Flutter analysis of bending-torsion coupling of aeroengine compressor blade with assembled clearance, Appl. Math. Model., 39 (2015), pp. 2539-2553.

[2] H. MA, Y. LU, Z. Y. WU, X. Y. TAI AND B. C. WEN, Vibration response analysis of a rotational shaft-disk-blade system with blade-tip rubbing, Int. J. Mech. Sci., 107 (2016), pp. 110-125.

[3] A. Rosen, Structural and dynamic behavior of pre-twisted rods and beams, Appl. Mech. Rev., 44 (1991), pp. 483-515.

[4] N. S. VYAS AND J. S. RAO, Equations of motion of a blade rotating with variable angular velocity, J. Sound. Vib., 156 (1992), pp. 327-336.

[5] S. M. LIN, The instability and vibration of rotating beams with arbitrary pre-twist and an elastically restrained root, J. Appl. Mech., 68 (2001), pp. 844-853.

[6] H. T. YOO, J. H. PARK AND J. PARK, Vibration analysis of rotating per-twisted blades, Comput. Struct., 79 (2001), pp. 1811-1819.

[7] N. K. ChANDIRAMANi, C. D. SHETE AND L. I. LiBReSCUB, Vibration of higher-order-shearable pre-twisted rotating composite blades, Int. J. Mech. Sci., 45 (2003), pp. 2017-2041.

[8] L. LibresCU, S. Y. OH, O. SONG AND H. S. KANG, Dynamics of advanced rotating blades made of functionally graded materials and operating in a high temperature field, J. Eng. Math., 61 (2008), pp. 1-16. 
[9] E. CARRERA, M. FILIPPI AND E. ZAPPINO, Free vibration analysis of rotating composite blades via carrera unified formulation, Compos. Struct., 106 (2013), pp. 317-325.

[10] M. H. YAO, Y. P. CHEN AND W. ZHANG, Nonlinear vibrations of blade with varying rotating speed, Nonlinear. Dyn., 68 (2012), pp. 487-504.

[11] H. H. YOO, S. SEO AND K. HUH, The effect of a concentrated mass on the modal characteristics of a rotating cantilever beam, P. I. Mech. Eng. C-J. Mech., 216 (2002), pp. 151-163.

[12] D. I. CHORTIS, D. S. VARELIS AND D. A. SARAVANOS, Prediction of material coupling effect on structural damping of composite beams and blades, Compos. Struct., 94 (2012), pp. 1646-1655.

[13] C. H. YANG AND S. C. HuANG, Coupling vibrations in rotating shaft-disk-blades system, J. Vib. Acoust., 129 (2007), pp. 48-57.

[14] Y. G. CHEN, J. Y. ZHAI AND Q. K. HAN, Vibration and damping analysis of the bladed disk with damping hard coating on blades, Aerosp. Sci. Technol., 58 (2016), pp. 248-257.

[15] S. SINA AND H. HADDADPOUR, Axial-torsional vibrations of rotating pre-twisted thin walled composites beams, Int. J. Mech. Sci., 80 (2014), pp. 93-101.

[16] Y. CHEN, J. ZHANG AND H. ZHANG, Free vibration analysis of rotating tapered Timoshenko beams via variational iteration method, J. Vib. Control., 39 (2015), pp. 754-765.

[17] S. K. SINHA AND K. E. TURNER, Natural frequencies of a pre-twisted blade in a centrifugal force field, J. Sound. Vib., 330 (2011), pp. 2655-2681.

[18] M. A. DOKAINISH AND S. RAWTANI, Vibration analysis of rotating cantilever plates, Int. J. Numer. Meth. Eng., 3 (1971), pp. 233-248.

[19] A. W. Leissa, J. C. MACbAIN AND R. E. KIELB, Vibrations of twisted cantilevered platessummary of previous and current studies, J. Sound. Vib., 96 (1984), pp. 159-173.

[20] M. S. QATU AND A. W. LEISSA, Vibration studies for laminated composite twisted cantilevered plates, Int. J. Mech. Sci., 33 (1991), pp. 927-940.

[21] C. W. Lim AND K. M. LIEW, Vibration of pretwisted cantilever trapezoidal symmetric laminates, Acta. Mech., 111 (1995), pp. 193-208.

[22] H. H. YOO, J. Y. KWAK AND J. CHUNG, Vibration analysis of rotating pre-twisted blades with a concentrated mass, J. Sound. Vib., 240 (2001), pp. 891-908.

[23] X. X. HU, T. SAKIYAMA AND K. ITAKURA, A new numerical procedure for free vibrations of pre-twisted plates, Arch. Appl. Mech., 72 (2002), pp. 330-341.

[24] S. F. XIAO AND B. CHEN, Dynamic behavior of thin rectangular plate attached to moving rigid, Appl. Math. Mech., 27 (2006), pp. 555-566.

[25] S. FARHADI AND S. H. HOSSEINI-HASHEMI, Aerolastic behavior of cantilevered rotating rectangular plates, Int. J. Mech. Sci., 53 (2011), pp. 316-328.

[26] J. SUn, L. KARI AND I. LOPEZ-ARTEAGA, A dynamic rotating blade model at an arbitrary stagger angle based on classical plate theory and the Hamiltons' principle, J. Sound. Vib., 332 (2013), pp. 1355-1371.

[27] L. LI AND D. G. ZHANG, Free vibration analysis of rotating functionally graded rectangular plates, Compos. Struct., 136 (2016), pp. 493-504.

[28] D. X. CAO, B. Y. LIU, M. H. YAO AND W. ZHANG, Free vibration analysis of a pre-twisted sandwich blade with thermal barrier coatings layers, Sci. China. Technol., 60 (2017), pp. 1-15.

[29] S. A. Fazelzadeh, P. MaleKZadeh, P. Zahedinejad AND M. Hosseini, Vibration analysis of functionally graded thin-walled rotating blades under high temperature supersonic flow using the differential quadrature method, J. Sound. Vib., 306 (2007), pp. 333-348.

[30] Z. KAZANCI, A review on the response of blast loaded laminated composite plates, Prog. Aerosp. Sci., 81 (2016), pp. 49-59.

[31] X. X. HU, T. SAKIYAMA, H. MATSUdA AND C. Morita, Fundamental vibration of rotating 
cantilever blades with pre-twist, J. Sound. Vib., 271 (2004), pp. 47-66.

[32] X. X. Hu, C.W. Lim, T. SAKIYAMA, Z. R. Li AND W. K. WANG, Free vibration of elastic helicoidal shells, Int. J. Mech. Sci., 47 (2005), pp. 941-960.

[33] S. K. SinHA AND K. E. TURNER, Natural frequencies of a pre-twisted blade in a centrifugal force field, J. Sound. Vib., 330 (2011), pp. 2655-2681.

[34] S. K. SINHA AND R. P. ZYLKA, Vibration analysis of composite airfoil blade using orthotropic thin shell bending theory, Int. J. Mech. Sci., 121 (2016), pp. 90-105.

[35] M. S. QATU, R. W. SUlLivan AND W. C. WANG, Recent research advances on the dynamic analysis of composite shells: 2000-2009, Compos. Struct., 93 (2010), pp. 14-31.

[36] J. Sun, I. L. ARTEAGA AND L. KARI, General shell model for a rotating pre-twisted blade, J. Sound. Vib., 332 (2013), pp. 5804-5820.

[37] A. K. NAYAK, R. A. SHENOI AND S. S. J. MOY, Transient response of composite sandwich plates, Compos. Struct., 64 (2004), pp. 249-267.

[38] K. GUPTA AND J. S. RAO, Torsional vibrations of pre-twisted cantilever plates, J. Mech. Design., 100 (1978), pp. 528-534.

[39] L. LIBRESCU AND A. NOSIER, Response of shear deformable elastic laminated composite panels to sonic boom and explosive blast loadings, Aiaa. J., 28 (1990), pp. 345-353.

[40] M. S. QATU AND A. W. LeISSA, Natural frequencies for cantilevered Doubly-Curved laminated composite shallow shells, Compos. Struct., 17 (1991), pp. 227-255.

[41] L. H. HE, C. W. Lim AND S. KitipORNCHAI, A non-discretized global method for free vibration of generally laminated fibre-reinforced pre-twisted cantilever plates, Comput. Mech., 26 (2000), pp. 197-207.

[42] A. BHIMARADDI, Large amplitude vibrations of imperfect antisymmetric angle-ply laminated plates, J. Sound. Vib., 162 (1999), pp. 457-470.

[43] A. NOSIR AND J. N. REDDY, A study of non-linear dynamic equations of higher-order deformation plate theories, Int. J. Nonlinear Mech., 26 (1991), pp. 233-249.

[44] P. N. BROWN, G. D. BYRNE AND A. C. HINDMARSH, VODE, a variable-coefficient ODE solver, SIAM. J. Sci. Stat. Comput., 10 (1989), pp. 1038-1051.

[45] A. C. HindmARSH, A systematized collection of ODE solvers, in: Stepleman R. S. et al., editors, Scientific Computing, IMACS Transactions on Scientific Computation, Amsterdam: NorthHolland, 1 (1983), pp. 55-64.

[46] K. RADHAKRISHNAN AND A.C. HINDMARSH, Description and use of LSODE, the Livermore solver for ordinary differential equations, LLNL report UCRL-ID-113855; (1993).

[47] P. N. BROWN AND A. C. HINDMARSH, Reduced storage matrix methods in stiff ODE systems, J. Comput. Appl. Math., 31 (1989), pp. 40-91.

[48] J. N. REDDY, Analysis of functionally graded plates, Int. J. Numer. Meth. Eng., 47 (2000), pp. 663-684.

[49] K. ZAFER AND M. ZAHIT, Nonlinear dynamic behavior of simply supported laminated composite plates subjected to blast load, J. Sound. Vib., 317 (2008), pp. 883-897.

[50] F. ALIJANI AND M. AMABILI, Nonlinear vibrations of laminated and sandwich rectangular plates with free edges, Part 1: Theory and numerical simulations, Compos. Struct., 105 (2013), pp. 422436. 